\title{
Evaluación de la calidad de los servicios en la Universidad Andina del Cusco, facultad de Ingeniería y arquitectura -escuela de Ingeniería industrial Evaluation of the Quality of Services at the Universidad Andina del Cusco, Faculty of Engineering and Architecture - School of Industrial Engineering
}

\author{
Isidro Castelán Herrera ${ }^{1,}{ }^{\doteq}$, Breezy Pilar Martínez Paredes ${ }^{1}$.
}

1 Universidad Andina del Cusco, Cusco, Perú.

” castelan_herrera@hotmail.com

\begin{abstract}
Resumen
El presente trabajo planteó una evaluación de la calidad de los servicios de la Universidad Andina del Cusco, Facultad de Ingeniería y Arquitectura, específicamente en la Escuela Profesional de Ingeniería Industrial, utilizando la herramienta Servqual (Service Quality) y los criterios de acreditación del "Instituto de calidad y acreditación de programas de computación, ingeniería y tecnología" (ICACIT), con la finalidad de mejorar los servicios de la calidad educativa e identificar las áreas críticas para mejora continua y aseguramiento de la calidad. La investigación se define como exploratoria-descriptiva, con un enfoque mixto, es decir, la integración de métodos cualitativos y cuantitativos, donde se utilizan técnicas e instrumentos de recolección, análisis e interpretación de datos, tales como muestreo aleatorio, cuestionarios y estadística descriptiva. El estudio se aplicó a estudiantes hombres y mujeres, de $1^{\circ}$ a $10^{\circ}$ semestre académicos, sin importar la edad. El análisis de los resultados de la percepción del nivel de la calidad se efectúa mediante el uso de paquetes estadísticos como Microsoft Excel y SPSS 22, los cuales ayudarán a obtener diversos valores de la percepción del nivel de calidad de los servicios que ofrece la universidad a los alumnos. Finalmente, la aplicación del cuestionario de los servicios de calidad fue realizada a través de la plataforma ERP UNIVERSITY, en el que se obtuvo una participación del $46 \%$ del alumnado matriculado en el semestre
\end{abstract}

Palabras clave: calidad, Servqual, Acreditación, Servicio, ICACIT

\begin{abstract}
This paper presents an evaluation of the quality of the services of the Andean University of Cusco, Faculty of Engineering and Architecture, specifically in the Professional School of Industrial Engineering, using the Servqual tool and the accreditation criteria of the "Institute of quality and accreditation of computer, engineering and technology programs "(ICACIT), with the purpose of improving educational quality services and identifying critical areas for continuous improvement and quality assurance. Research is defined as exploratory-descriptive, with a mixed approach, that is, the integration of qualitative and quantitative methods, where techniques and instruments for data collection, analysis and interpretation are used, such as random sampling, questionnaires and descriptive statistics. The study was applied to male and female students, from 1st to 10 th academic semester, regardless of age. The analysis of the results of the perception of the level of quality is carried out through the use of statistical packages such as Microsoft Excel and SPSS 22, which will help to obtain different values of the perception of the level of quality of the services offered by the university to the students. Finally, the quality services questionnaire was applied through the ERP UNIVERSITY platform, in which a $46 \%$ participation of the students enrolled in the semester was obtained
\end{abstract}

Keywords: Labor market transitions, pooled logit, two-sector search model, search and matching theory

Citar como: Herrea, IC., Paredes, BPM. (2019). Evaluación de la calidad de los servicios en la universidad andina del cusco, facultad de ingeniería y arquitectura -escuela de ingeniería industrial. Rev Yachay, 8(1), 535-543.

Recibido: 1-09-2019; Aceptado 07-12-2019; Publicado: 12-12-2019

\section{Introducción}

El empleo del concepto de calidad, se suele imaginar como un excelente producto o servicio, que cumple o rebasa las expectativas de clientes y usuarios. Si un producto excede las expectativas, a eso se le considera calidad. Entonces, la calidad es algo intangible que se basa en la percepción (Besterfield, 2009). La American Society for Quality (ASQ, 1983) define calidad como un vocablo subjetivo ya 
que cada persona, empresa o institución tendrá una definición de acuerdo a lo que requiera según requisitos o exigencias. En la aplicación técnica e industrial, la calidad se define como las características de un producto, servicio u objeto que inciden en su capacidad para satisfacer las necesidades manifiestas o supuestas, y que un producto o servicio está libre de deficiencias (Daniels \& Dave, 2007).

Como se sabe, a lo largo de la historia se han realizado diversas contribuciones y modificaciones al concepto de calidad, hoy en día el concepto de calidad más utilizado debido a su aplicación es el que ha establecido la Norma ISO 9000:2015, la cual describe a la calidad como el grado en el que un conjunto de características inherentes de un objeto cumple con los requisitos (ISO, 2015). A partir de lo anterior se tiene que la calidad de la prestación del servicio y la satisfacción del cliente han sido desde hace tiempo identificadas como elementos clave del servicio (Heskett, Passer, y Schlesinger, 1997) es por ello que esta investigación se centra en averiguar cuál es la percepción de calidad que tienen los alumnos de la Escuela Profesional de Ingeniería Industrial, así como los conceptos sobre la calidad de servicio, es así que, Rosander (1992) ha definido al servicio como un sistema en el cual el trabajo se efectúa de una forma preestablecida y su propósito es satisfacer las preferencias, gustos y necesidades de los clientes. Payne (1996) define al servicio como una actividad con cierta intangibilidad asociada, lo que implica la interacción con clientes o con alguna propiedad de su posesión; por lo tanto, la producción de un servicio alcanza estar o no intrínsecamente asociado con un producto físico.

Para Kotler y Armstrong (2008) los servicios son una forma de producto el cual consiste en actividades, beneficios o satisfacciones puestos a la venta y son fundamentalmente impalpables, ya que no tienen como consecuencia la obtención de la pertenencia de la propiedad. De lo anterior se tiene que la norma ISO 9000:2015 en el numeral 3.7.7., el servicio es la salida de una organización con al menos una actividad, necesariamente llevada a cabo entre la organización y el cliente. En consecuencia, este estudio de investigación realizado es con el objetivo de evaluar el nivel de calidad de servicios en la escuela profesional de Ingeniería Industrial en la Facultad de Ingeniería y Arquitectura, de la Universidad Andina del Cusco, mediante la aplicación de la herramienta SERVQUAL, con la finalidad de mejorar los servicios de la calidad educativa e identificar las áreas críticas para mejora continua y aseguramiento de la calidad. Finalmente, el aporte que se tendrá con la evaluación de los niveles de percepción de la calidad por parte de los alumnos es optimizar el trabajo que se realiza en el campo de la educación y sus procesos inherentes, la construcción de la metodología de evaluación permitirá visualizar las percepciones que tiene el alumno sobre los servicios que se le ofrecen.

\title{
Calidad de servicio
}

La calidad de servicio viene ofrecida por la cercanía entre el servicio esperado y el servicio percibido; es decir, el servicio que un cliente desea recibir y el servicio que distingue en el momento que se encuentra en el sistema o local del proveedor. Entonces se define a la calidad de servicio como la extensión de la discrepancia que exista entre expectativas de los clientes y sus percepciones (Zeithaml, Parasuraman y Berry, 1990). Grönroos (1994) admite la determinación de la calidad en los servicios la cual debe estar basada fundamentalmente en las percepciones que los clientes tienen del servicio. Entonces se establece la definición de calidad de servicio la cual es el juicio global del consumidor acerca de la excelencia o superioridad global del producto (Camisón et al., 2006).

Hoy en día existen diversas metodologías para conocer el grado de satisfacción de los clientes, por lo que las empresas, organizaciones e instituciones pueden utilizar diversas técnicas y prácticas tales como: formularios de quejas y reclamaciones, buzones de sugerencias, la pseudocompra, captura del número de clientes perdidos, cuestionarios de satisfacción. Esta última herramienta es la más utilizada ya que permite obtener la información directamente de los clientes. Es por ello que, los diversos trabajos de investigación de los autores Parasuraman, Zeithaml y Berry (1990) les permitieron desarrollar el modelo SERVQUAL que está formado por cinco dimensiones: Elementos tangibles, Fiabilidad, Capacidad de respuesta, Seguridad y Empatía. Las instituciones de educación deben ofrecer servicios de calidad por lo que se han desarrollado modelos de acreditación que permiten evaluar la calidad de la educación y los servicios que estas ofrecen a sus clientes y/o alumnos. Tal es el caso de ICACIT el cual es una agencia acreditadora especializada en programas de formación profesional en computación, ingeniería y tecnología. ICACIT promueve la mejora continua de la calidad educativa de los programas, garantizando que estos cumplan con los más altos estándares internacionales todo lo anterior mediante criterios que aseguren que los graduados estén listos para ejercer su profesión (ICACIT, 2019).

\author{
Rev. Yachay volumen (8) Número (1), enero-diciembre 2019
}




\begin{tabular}{|c|c|}
\hline Criterio & Descripción \\
\hline Estudiantes & $\begin{array}{l}\text { Toma en consideracion el proceso de admisión a la carrera, la evaluación del rendimeinto } \\
\text { de los estudiantes, el asesoramiento de los estudiantes, las actividades extracurriculares y } \\
\text { los requisitos vigentes de la universidad. }\end{array}$ \\
\hline $\begin{array}{l}\text { Objetivos } \\
\text { Educacionales } \\
\text { del Programa }\end{array}$ & $\begin{array}{l}\text { Son declaraciones generales que describen lo que se espera que los graduados logren algunos } \\
\text { años despues de la graduación. }\end{array}$ \\
\hline $\begin{array}{l}\text { Resultados del } \\
\text { Estudiante }\end{array}$ & $\begin{array}{l}\text { El criterio hace referencia a las habilidades, conocimientos y comportamientos del } \\
\text { estudiante. ICACIT establece doce Resultados del Estudiante los cuales son: Conocimentos } \\
\text { de Ingeniería, Experimentación, Diseño y desarrollo de soluciones, Trabajo individual y en } \\
\text { equipo, Análisis de problemas, Ética, Comuniación, Medio ambiente y sostenibilidad, } \\
\text { Aprendizaje permanente, El ingeniero y la sociedad, Uso de herramientas modernas y por } \\
\text { ultimo, Gestión de proyectos. }\end{array}$ \\
\hline $\begin{array}{l}\text { Mejora } \\
\text { Continua }\end{array}$ & $\begin{array}{l}\text { Se considera la base del modelo de ICACIT y exige que el programa utilice regularmente } \\
\text { procesos documentados y apropiados en el Assesment y la evaluación del grado en el que } \\
\text { los Resultados del Estudiante están siendo logrados. }\end{array}$ \\
\hline $\begin{array}{l}\text { Plan de } \\
\text { Estudios }\end{array}$ & $\begin{array}{l}\text { Es la consistencia que debe de tener el Plan de estudios con los Resultados del Estudiante y } \\
\text { los Objetivos Educacionales del Programa. }\end{array}$ \\
\hline $\begin{array}{l}\text { Cuerpo de } \\
\text { Profesores }\end{array}$ & $\begin{array}{l}\text { El Programa Académico debe demostrar que cuenta con un número adecuado de profesores } \\
\text { para que se cubran las áreas del Plan de Estudios, además de las funciones de orientación a } \\
\text { estudiantes, interacción con la insdustria, Assessment y mejora continua. }\end{array}$ \\
\hline Instalaciones & $\begin{array}{l}\text { La infraestuctura y equipos empleados por el Programa Académico deben de ser adecuados } \\
\text { para el logro de los Resulatdos del Estudiante. }\end{array}$ \\
\hline $\begin{array}{l}\text { Apoyo } \\
\text { Institucional }\end{array}$ & $\begin{array}{l}\text { Aquí se debe demostrar que los recursos y el liderazgo institucional son suficientes y } \\
\text { correctos para asegurar la calidad y la continuidad del Programa Académico. }\end{array}$ \\
\hline $\begin{array}{l}\text { Investigación e } \\
\text { Innovación }\end{array}$ & $\begin{array}{l}\text { El Programa Académico debe regular y asegurar la calidad de las investigaciones } \\
\text { realizadas por docentes y estudiantes, ademas de ello, se debe procurar brindar los medios } \\
\text { para promover la investigación. }\end{array}$ \\
\hline
\end{tabular}

Fuente: Extraído de Chávez, Madrid, et al. (2018) e ICACIT (2019)

\section{Materiales y métodos}

De acuerdo a Hernández et al., (2010), el aspecto de la metodología que se utilizó en este trabajo de investigación tiene un enfoque cuantitativo, el cual se fundamenta en un esquema deductivo, este se basa en el cálculo de la desviación estándar, la media aritmética de percepciones de los alumnos de la Escuela Profesional de Ingeniería Industrial (EPII), con la finalidad de determinar las áreas de oportunidad a mejorar por la universidad, la facultad y la EPII. Los cálculos se trabajaron utilizando la aplicación del software Excel 2016 (hojas de cálculo) del paquete de Microsoft Office en conjunto con el paquete estadístico SPSS 22.0 (Statistical Package for the Social Sciences).

De acuerdo a Hernández et al. (2010) esta investigación tiene una orientación descriptiva, porque evalúa o mide diversos aspectos del fenómeno a investigar. Es decir, este estudio procura evaluar el nivel de percepción y satisfacción de los servicios que brinda la Universidad. De igual manera, este trabajo de investigación es de tipo transversal no experimental, debido a que, en los estudios realizados, una investigación no experimental solo procura observar los fenómenos tal y como se dan en su ambiente natural para, a continuación, estudiarlos sin perturbar alguna variable.

Para la realización de este proyecto de investigación se tomaron como sujetos de estudio a los estudiantes/alumnos de la EPII en la Facultad de Ingeniería y Arquitectura, puesto que las percepciones referentes a la calidad del servicio son evaluaciones que realizan exclusivamente los alumnos.

Para el presente trabajo se tomó una muestra aleatoria representativa $(\mathrm{n}=366)$ del total de los alumnos que reciben los servicios que proporciona la Universidad durante el semestre 2019-I. El tamaño de la muestra fue obtenido utilizando la fórmula estadística para 
poblaciones finitas, Para la medición de las variables de estudio se utilizó el cuestionario diseñado mediante Servqual y los Criterios del Modelo ICACIT, que se muestra en la Tabla 2. De este se obtienen las percepciones del servicio de calidad que ofrece la Universidad.

Tabla 2

Cuestionario SERVQUAL adaptado a criterios de ICACIT (Percepciones)

\begin{tabular}{|c|c|c|}
\hline CRITERIO & No & ÍTEM \\
\hline Estudiantes & $\begin{array}{l}1 \\
2 \\
3 \\
4 \\
5\end{array}$ & $\begin{array}{l}\text { Monitoreo del progreso de los estudiantes. } \\
\text { Evaluación permanente por lo docentes. } \\
\text { Conocimientos suficientes para responder a las cuestionamientos de los alumnos. } \\
\text { Atención específica. } \\
\text { Comunicación fluida entre alumnos y profesores. }\end{array}$ \\
\hline $\begin{array}{l}\text { Objetivos } \\
\text { Educacionales del } \\
\text { Programa (OEP) }\end{array}$ & $\begin{array}{l}6 \\
7 \\
8\end{array}$ & $\begin{array}{l}\text { Comunicación y divulgación de la misión. } \\
\text { Comunicación de los OEP a los alumnos. } \\
\text { Fomento de la educación integral de sus estudiantes. }\end{array}$ \\
\hline $\begin{array}{l}\text { Resultados del } \\
\text { Estudiante }\end{array}$ & $\begin{array}{c}9 \\
10 \\
11\end{array}$ & $\begin{array}{l}\text { Logro de los conocimientos requeridos. } \\
\text { Formación de profesionales competentes. } \\
\text { Desarrollo de sólida base científica y humanista en el estudiante. }\end{array}$ \\
\hline Mejora Continua & $\begin{array}{l}12 \\
13 \\
14 \\
15 \\
16 \\
17 \\
18 \\
19\end{array}$ & $\begin{array}{l}\text { Servicio bien a la primera. } \\
\text { Servicio en el tiempo programado. } \\
\text { Conclusión de la realización del servicio que se presta. } \\
\text { Servicio rápido a sus usuarios. } \\
\text { Disposición a ayudar a los usuarios. } \\
\text { Seguridad en las relaciones con la universidad (pagos, atención recibida, etc.). } \\
\text { Excepción de errores en el servicio. } \\
\text { Desarrollo de metodologías nuevas y novedosas para el desarrollo en el aula. }\end{array}$ \\
\hline Plan de Estudios & $\begin{array}{l}20 \\
21 \\
22 \\
23 \\
24\end{array}$ & $\begin{array}{l}\text { El material entregado útil y atractivo para los estudiantes. } \\
\text { Se concluyen los sílabos durante el desarrollo de un curso. } \\
\text { Se respetan estrictamente el desarrollo de los sílabos. } \\
\text { Los sílabos se modifican durante el desarrollo de un curso. } \\
\text { Los horarios son convenientes para todos sus usuarios. }\end{array}$ \\
\hline $\begin{array}{l}\text { Cuerpo de } \\
\text { Profesores }\end{array}$ & $\begin{array}{l}25 \\
26 \\
27 \\
28 \\
29 \\
30\end{array}$ & $\begin{array}{l}\text { Personal con buena apariencia. } \\
\text { Disponibilidad para responder a las preguntas de los alumnos. } \\
\text { Se transmite confianza a sus usuarios. } \\
\text { Amabilidad con sus usuarios. } \\
\text { Actualización permanentemente. } \\
\text { Expectativas de enseñanza. }\end{array}$ \\
\hline Instalaciones & $\begin{array}{l}31 \\
32 \\
33 \\
34\end{array}$ & $\begin{array}{l}\text { Equipos modernos e instalaciones físicas atractivas. } \\
\text { Infraestructura suficiente. } \\
\text { Espacios suficientes de recreación. } \\
\text { Planes de mantenimiento y actualización de las instalaciones. }\end{array}$ \\
\hline $\begin{array}{l}\text { Apoyo } \\
\text { Institucional }\end{array}$ & $\begin{array}{l}35 \\
36 \\
37 \\
38\end{array}$ & $\begin{array}{l}\text { Cuando un usuario tiene un problema, se muestra interés en resolverlo. } \\
\text { Atención personalizada a sus usuarios. } \\
\text { Preocupación por los intereses y necesidades de sus usuarios. } \\
\text { Se comprenden las necesidades específicas de sus usuarios. }\end{array}$ \\
\hline $\begin{array}{l}\text { Investigación e } \\
\text { Innovación }\end{array}$ & $\begin{array}{l}41 \\
42 \\
43 \\
44 \\
45 \\
46\end{array}$ & $\begin{array}{l}\text { Se realizan trabajos de investigación para la obtención del grado académico, asegurando la rigurosidad, } \\
\text { pertinencia y calidad. } \\
\text { Se promueve la publicación de los trabajos de investigación de sus profesores y su respectiva socialización } \\
\text { dentro de la institución. } \\
\text { Se realizan concursos de investigación de los alumnos. } \\
\text { Los docentes invitan a trabajos de investigación a los alumnos. } \\
\text { Los trabajos de investigación se desarrollan multidisciplinariamente. } \\
\text { Los docentes desarrollan metodologías novedosas para la investigación. } \\
\text { Los círculos de investigación cumplen con las expectativas de los alumnos. } \\
\text { Los círculos de investigación cumplen con las expectativas de los docentes. }\end{array}$ \\
\hline
\end{tabular}

Fuente: Adaptado de ICACIT (2019) y Zeithaml, Parasuraman y Berry (1993).

Del cuestionario de la Tabla 1, se obtienen las percepciones del servicio de calidad que recibe el alumno de la universidad, lo cual permite identificar las áreas de oportunidad. 


\section{Resultados}

Los resultados estadísticos de los datos generales obtenidos del cuestionario, permiten determinar los valores de las percepciones que tienen los alumnos del servicio de la Universidad. La validación del constructo manifestó una medida de adecuación de KMO de 0.969. La Prueba de esfericidad de Bartlett para el cuestionario mostró un valor significativo p =0,000. En el alfa de Cronbach se obtuvo un valor de 0.974 para las percepciones. En la Tabla 3, se presentan los resultados de las encuestas aplicadas a los alumnos de la EPII en la Facultad de Ingeniería y Arquitectura de la Universidad Andina del Cusco, siendo un total de 371 encuestados, con las variables de Edad, Sexo y Semestre. En la Tabla 4, se describen los estadísticos de acuerdo al Sexo de los alumnos.

Tabla 3

Estadísticos generales

\begin{tabular}{lccc}
\hline & Edad & Sexo & Semestre \\
\hline N Válido & 371 & 371 & 371 \\
Media & 21.58 & $\mathrm{H}$ & 5.2129 \\
Mediana & 21.00 & - & 5.0000 \\
Moda & $20^{\mathrm{a}}$ & $\mathrm{H}$ & 3.00 \\
Rango & 23 & - & 9.00 \\
Mínimo & 16 & - & 1.00 \\
Máximo & 39 & - & 10.00 \\
\hline
\end{tabular}

a. Existen múltiples modos. Se muestra el valor más pequeño.

Tabla 4

\begin{tabular}{llcccc} 
Sexo & & & & \\
& Sexo & Frecuencia & Porcentaje & \% válido & \% acumulado \\
\hline Válido & Masculino & 228 & 61.5 & 61.5 & 61.5 \\
& Femenino & 143 & 38.5 & 38.5 & 100.0 \\
& Total & 371 & 100.0 & 100.0 & \\
\hline
\end{tabular}

Fuente: datos obtenidos. SPSS 22.

De la Tabla 4, se aprecian los resultados de porcentajes de participación por sexo de los alumnos encuestados, teniendo un total de 371 , de los cuales 228 son masculinos que representan el $61.46 \%$ y 143 femeninas que representan el 38.54\%. A continuación, en la Tabla 5 , se exponen los datos de los encuestados por semestre que cursan durante la aplicación del cuestionario.

Tabla 5

\begin{tabular}{cccccc}
\multicolumn{2}{l}{ Semestre } \\
\multicolumn{2}{c}{ Semestre } & Frecuencia & Porcentaje & \% válido & \%acumulado \\
\hline Válido & 1 & 45 & 12.1 & 12.1 & 12.1 \\
& 2 & 30 & 8.1 & 8.1 & 20.2 \\
& 3 & 46 & 12.4 & 12.4 & 32.6 \\
& 4 & 45 & 12.1 & 12.1 & 44.7 \\
5 & 35 & 9.4 & 9.4 & 54.2 \\
6 & 37 & 10.0 & 10.0 & 64.2 \\
7 & 43 & 11.6 & 11.6 & 75.7 \\
\hline & 28 & 7.5 & 7.5 & 83.3 \\
& 31 & 8.4 & 8.4 & 100.0 \\
\hline
\end{tabular}

Fuente: datos obtenidos. SPSS 22. 


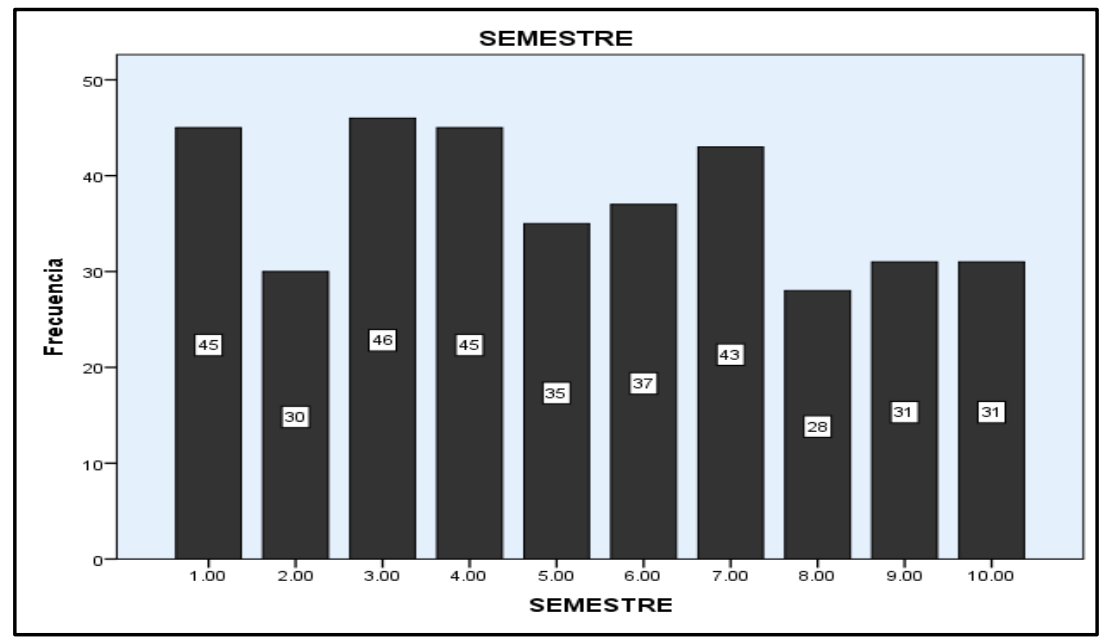

Figura 2. Semestre. Fuente: Elaboración propia de los datos obtenidos con SPSS 22.

En la Tabla 5 y la Figura 2 se muestran los resultados de participación de los alumnos encuestados por semestre que cursan teniendo la mayor participación con alumnos de tercer semestre con $12.4 \%$, primero y cuarto semestre con $12.1 \%$; además, alumnos de noveno y décimo semestre tuvieron una participación del $8.4 \%$

En la Tabla 6 se describe el nivel de calidad percibido por los alumnos de la Escuela Profesional de Ingeniería Industrial en la Facultad de Ingeniería y Arquitectura.

Tabla 6

Calidad de servicio por alumno

\begin{tabular}{|c|c|c|c|c|}
\hline Valor & $\begin{array}{l}\text { Descripción de } \\
\text { Nivel de Calidad }\end{array}$ & Frecuencia & $\%$ & $\begin{array}{c}\% \\
\text { acumulado }\end{array}$ \\
\hline 1 & Pésimo & 12 & $3 \%$ & $3 \%$ \\
\hline 2 & Malo & 23 & $6 \%$ & $9 \%$ \\
\hline 3 & Regular & 142 & $38 \%$ & $48 \%$ \\
\hline 4 & Bueno & 161 & $43 \%$ & $91 \%$ \\
\hline \multirow[t]{2}{*}{5} & Excelente & 33 & $9 \%$ & $100 \%$ \\
\hline & Total & 371 & $100 \%$ & \\
\hline
\end{tabular}

Fuente: Elaboración propia de los datos obtenidos.

En la Tabla 6, Figura 3 y 4, se muestran las valoraciones de la calidad que hacen los alumnos de la Escuela Profesional de Ingeniería Industrial por frecuencias, siendo que el $43 \%$ de los alumnos encuestados perciben una calidad buena, mientras que solo el $3 \%$ perciben una calidad pésima. 


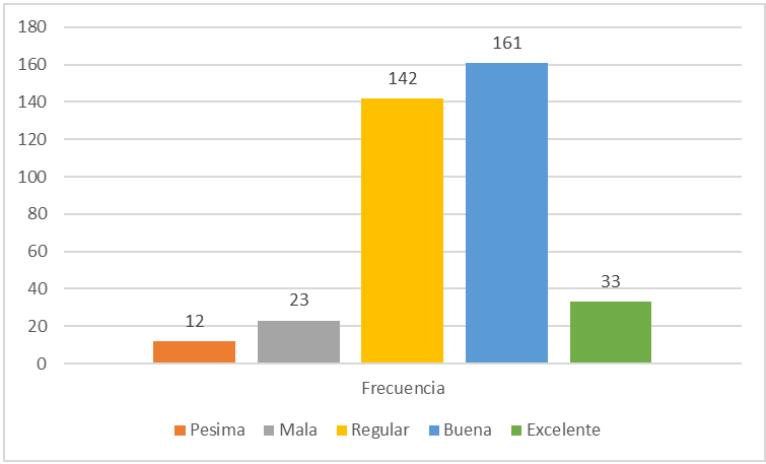

Figura 3. Calidad de servicio por alumnos.

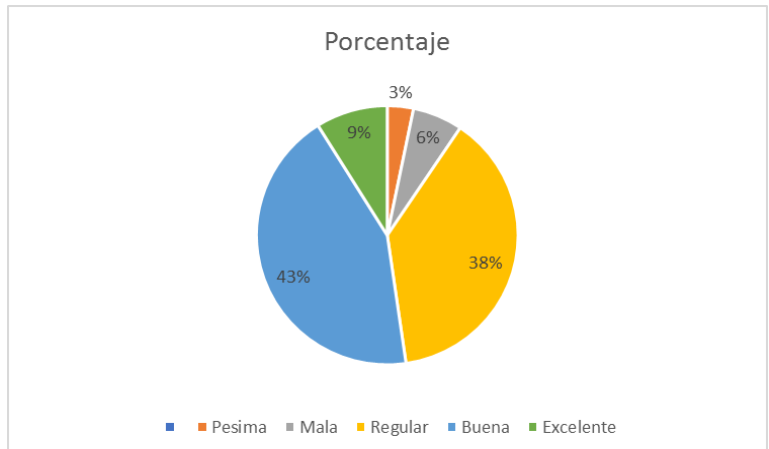

Figura 4. Calidad de servicio por alumnos en porcentaje.

Para el análisis descriptivo del cuestionario se tomaron las medidas estadísticas de tendencia central y dispersión con relación a las percepciones que tienen los alumnos del servicio que les brinda la universidad, las cuales se muestran a continuación en las Tablas 7 y 8.

Tabla 7.

Medidas de tendencia central y de dispersión del cuestionario.

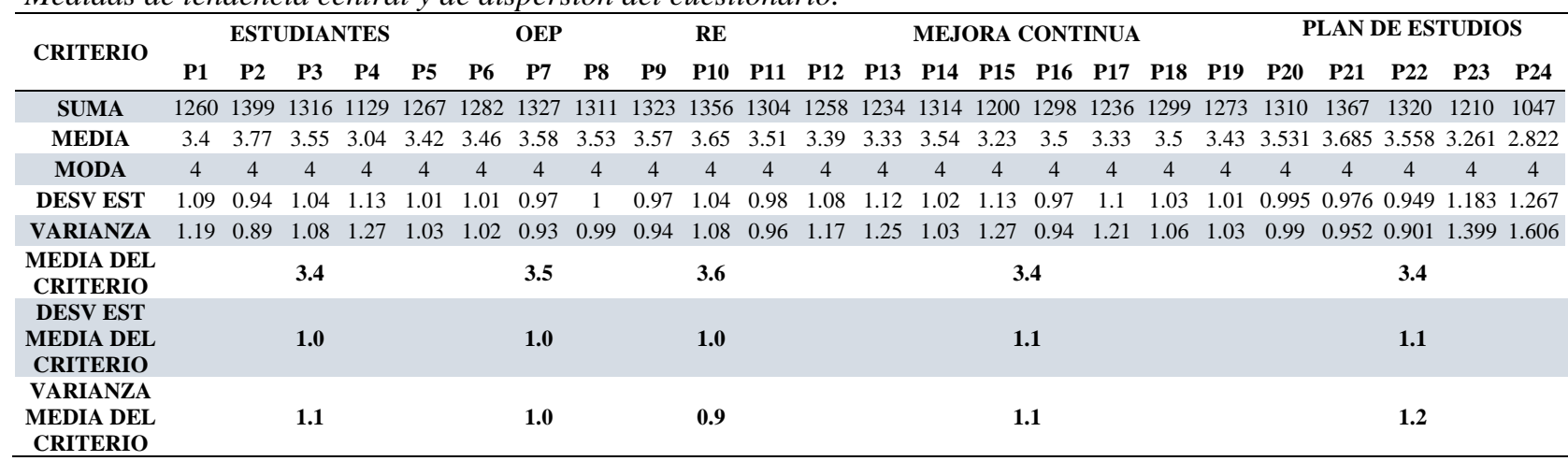

Tabla 8.

Medidas de tendencia central y de dispersión del cuestionario (Cont.)

\begin{tabular}{|c|c|c|c|c|c|c|c|c|c|c|c|c|c|c|c|c|c|c|c|c|c|c|c|}
\hline \multirow[t]{2}{*}{ CRITERIO } & \multicolumn{6}{|c|}{ CUERPO DE PROFESORES } & \multicolumn{4}{|c|}{ INSTALACIONES } & \multicolumn{4}{|c|}{$\begin{array}{c}\text { APOYO } \\
\text { INSTITUCIONAL }\end{array}$} & \multicolumn{8}{|c|}{ INVESTIGACIÓN E INNOVACIÓN } & \multirow[t]{2}{*}{ GLOBAL } \\
\hline & $\mathbf{P 2 5}$ & $\mathbf{P 2 6}$ & $\mathbf{P 2 7}$ & $\mathbf{P 2 8}$ & $\mathbf{P 2 9}$ & P30 & P31 & P32 & P33 & P34 & P35 & P36 & P37 & P38 & P39 & $\mathbf{P 4 0}$ & P41 & $\mathbf{P 4 2}$ & $\mathbf{P 4 3}$ & P44 & $\mathbf{P 4 5}$ & P46 & \\
\hline SUMA & 1237 & 1232 & 1264 & 1286 & 1309 & 1257 & 1244 & 1173 & 1152 & 1230 & 1155 & 1252 & 1196 & 1232 & 1337 & 1307 & 1364 & 1315 & 1295 & 1258 & 1209 & 1209 & 1264.20 \\
\hline MEDIA & 3.334 & 3.321 & 3.407 & 3.466 & 3.528 & 3.388 & 3.35 & 3.16 & 3.11 & 3.32 & 3.113 & 3.375 & 3.224 & 3.321 & 3.6 & 3.52 & 3.68 & 3.54 & 3.49 & 3.39 & 3.26 & 3.26 & 3.41 \\
\hline MODA & 4 & 4 & 4 & 4 & 4 & 4 & 4 & 4 & 4 & 4 & 3 & 4 & 4 & 4 & 4 & 4 & 4 & 4 & 4 & 4 & 4 & 3 & 3.96 \\
\hline DESV EST & 0.982 & 1.033 & 1.018 & 1.006 & 0.987 & 1.024 & 1.12 & 1.21 & 1.26 & 1.06 & 1.075 & 0.982 & 1.048 & 0.971 & 0.97 & 1 & 0.9 & 1 & 0.96 & 0.94 & 0.99 & 0.95 & 1.03 \\
\hline VARIANZA & 0.964 & 1.067 & 1.037 & 1.012 & 0.974 & 1.049 & 1.26 & 1.45 & 1.59 & 1.12 & 1.155 & 0.965 & 1.098 & 0.943 & 0.93 & 1 & 0.81 & 0.99 & 0.92 & 0.89 & 0.98 & 0.91 & 1.07 \\
\hline $\begin{array}{c}\text { MEDIA } \\
\text { DEL } \\
\text { CRITERIO }\end{array}$ & \multicolumn{6}{|c|}{3.4} & \multicolumn{4}{|c|}{3.2} & \multicolumn{4}{|c|}{3.3} & \multicolumn{8}{|c|}{3.5} & 3.41 \\
\hline $\begin{array}{l}\text { DESV EST } \\
\text { MEDIA } \\
\text { DEL } \\
\text { CRITERIO }\end{array}$ & \multicolumn{6}{|c|}{1.0} & \multicolumn{4}{|c|}{1.2} & \multicolumn{4}{|c|}{1.0} & \multicolumn{8}{|c|}{1.0} & 1.03 \\
\hline $\begin{array}{l}\text { VARIANZA } \\
\text { MEDIA } \\
\text { DEL } \\
\text { CRITERIO } \\
\end{array}$ & \multicolumn{6}{|c|}{1.0} & \multicolumn{4}{|c|}{1.4} & \multicolumn{4}{|c|}{1.0} & \multicolumn{8}{|c|}{0.9} & 1.07 \\
\hline
\end{tabular}


Por lo anterior, se tiene que la media global de la percepción es de 3.41, lo que indica que el alumno percibe un nivel de calidad bueno con lo que espera del servicio que le ofrece la universidad, en especial en la Facultad de Ingeniería y Arquitectura, y en particular a la Escuela Profesional de Ingeniería Industrial, todo ello con desviación estándar del 1.03 que indica cuanto están dispersos los valores con respecto a la media.

En la Figura 5, se observa que el criterio de Resultados del Estudiante (RE) es el de mayor media con un valor de 3.6 y el criterio de Instalaciones tiene la menor media (valor de 3.2) de los nueve criterios que se han considerado para el estudio. En la Figura 6 se muestran los resultados de la percepción del nivel de calidad por sexo, teniendo que las mujeres muestran una mayor percepción del nivel de la calidad de los servicios de la universidad.

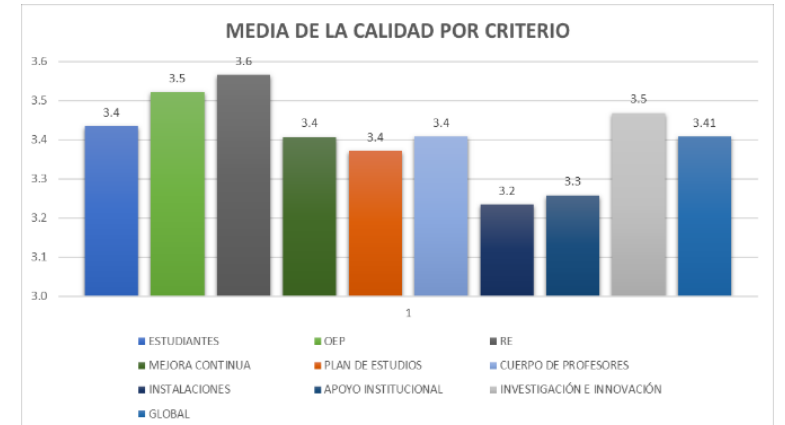

Figura 5. Media de la Calidad por Criterio.

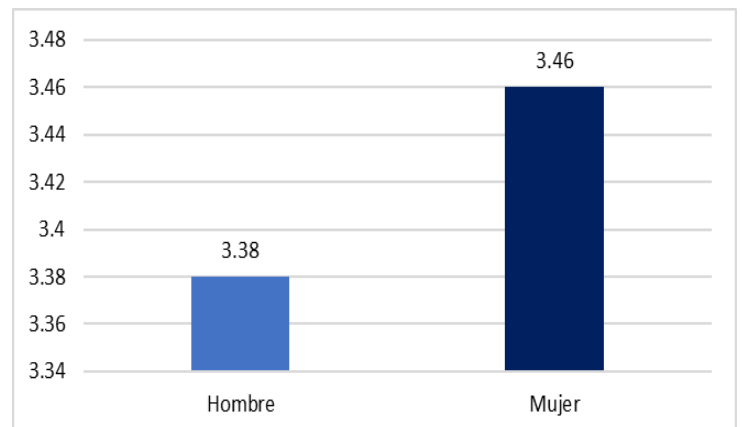

Figura 6. Media del Nivel de calidad por Sexo.

\section{Discusión}

El objetivo de este proyecto de investigación fue evaluar el nivel de calidad de los servicios en la Escuela Profesional de Ingeniería Industrial en la Facultad de Ingeniería y Arquitectura, de la Universidad Andina del Cusco, mediante la aplicación de la herramienta Servqual, con la finalidad de mejorar los servicios de la calidad educativa e identificar las áreas críticas para mejora continua y aseguramiento de la calidad.

Parasuraman et al. (1988) afirmaron que Servqual establecía cinco dimensiones para obtener la calidad del servicio, este estudio no confirma la estructura de las cinco dimensiones del instrumento, al igual que Guzmán y Cárcamo, (2014) donde reiteran la existencia de brechas en la mayoría de las dimensiones consideradas por los investigadores en los casos de estudio. Los resultados obtenidos no tienen un valor de comparación previo en un estudio debido a que se utilizaron el Servqual y los Criterios de Acreditación de Programas de Ingeniería de ICACIT, para desarrollar un instrumento que contenga los criterios que se ajusten al problema planteado; por tanto, de manera general se tiene una evaluación de la percepción de 3.41 como media global, lo que indica que se tiene una calidad buena en los servicios que se ofrecen en la Universidad. Sin embargo, ello no significa que se está realizando un buen servicio ya que en los valores de las escalas Likert el valor de tres representa Indiferencia o un nivel de calidad regular, por parte del alumno con respecto al servicio que está recibiendo.

En los resultados del estudio se observa que el criterio de Resultados del Estudiante (RE) es el de mayor media con un valor de 3.6 y el criterio de Instalaciones tiene la menor media (valor de 3.2) de los nueve criterios que se han considerado para el estudio. De igual forma los resultados de la percepción del nivel de calidad por sexo, se tiene que las mujeres muestran una mayor percepción del nivel de la calidad de los servicios de la universidad con un valor de 3.46, mientras que los hombres valoran la calidad de los servicios de la universidad con un valor medio de 3.38 .

Con este estudio la evaluación del nivel de la calidad de servicio fue posible realizar un análisis de las percepciones que se tienen por parte de alumnos de la Escuela de Ingeniería Industrial, en la Facultad de Ingeniería y Arquitectura, de la Universidad Andina del Cusco, ya que se obtuvo una valoración cuantitativa y cualitativa del nivel de calidad de los servicios siendo esta de 3.41 que indica que existe un nivel de calidad bueno.

\section{Conclusiones}

Primera. En el presente estudio se observa que mediante el procedimiento que se siguió durante esta investigación se logró cumplir el objetivo general planteando inicialmente, el cual consiste en evaluar el nivel de calidad de los servicios en la Escuela Profesional de Ingeniería Industrial en la Facultad de Ingeniería y Arquitectura, de la Universidad Andina del Cusco, mediante la aplicación de la herramienta Servqual, con la finalidad de mejorar los servicios de la calidad educativa e identificar las áreas críticas para la mejora continua y aseguramiento de la calidad, ya que se consiguió establecer los métodos con los cuales se analiza el nivel de calidad de servicio que es ofrecido por la UAC, y con ello se identificaron los criterios en la que los estudiantes se mostraron insatisfechos durante el servicio recibido. 
En el formato de evaluación aplicado a los alumnos fue bien recibido, ya que se presentó de manera electrónica mediante la plataforma ERP UNIVERSITY, y se obtuvo un total de participación de 371 alumnos de 807 que se encuentran inscritos en el semestre en el que se desarrolló el estudio, esto representa un $46 \%$ de la participación de la matrícula de la Escuela Profesional de Ingeniería Industrial. Los objetivos específicos planteados también se han logrado ya que se pudo diagnosticar la percepción del nivel de la calidad por parte de alumnos en cuanto a servicio, procesos e instalaciones por medio de la aplicación del Servqual y los Criterios de Acreditación de Programas de Ingeniería de ICACIT.

Segunda. El análisis de los resultados de la percepción del nivel de la calidad mediante el uso de la estadística a fin de permitir la medición de la calidad, se llevó a cabo mediante el uso de paquetes estadísticos como Microsoft Excel y SPSS 22, los cuales permitieron obtener los valores de las percepciones del nivel de calidad de los servicios que ofrece la universidad a los alumnos, teniendo como valor medio 3.41 , lo que indica que se tiene una calidad buena en los servicios que se ofrecen.

\section{Referecias bibliografía}

ASQ. (1983). ASQ Statistics División. Glossary and Tables for Statistical Quality Control. Milwaukee, Wis: American Society for Quality.

Besterfield, D. H. (2009). Control de calidad (Octava ed.). México: Pearson Educación.

Camisón, C., Cruz, S., \& González, T. (2006). Gestión de la Calidad: conceptos, enfoques, modelos y sistemas. Madrid, España: Pearson Educación, S. A.

Cuatrecasas, L. (2010). Gestión Integral de la Calidad: Implantación, Control y Certificación. Barcelona, España: Profit Editorial Inmobiliaria, S.L.

Daniels, S. E., y Dave N. (2007). Quality Glossary. Quality Progress, 39-59.

Evans, J. R. (2008). Administración y control de la calidad. México, D.F., México: Cengage Learning Editores, S.A. de C.V

Grönroos, C. (1994). Marketing y gestión de servicios. Madrid: Díaz de Santos.

Guzmán, L. A., y Cárcamo, S. M. (2014). La evaluación de la calidad en el servicio: caso de estudio "Restaurant Familiar Los Fresnos”. Acta Universitaria, 35-49. DOI:10. 15174.au.2014.503

ICACIT. (2019). Criterios para la Acreditación de Programas de Ingeniería.
ISO. (2015). Sistemas de gestión de la calidadFundamentos y vocabulario. Ginebra,

Suiza.

Malhotra, N. K. (2008). Investigación de Mercados (Quinta ed.). México: Pearson Educación.

Mejías, A., Villegas, D., y Maneiro, N. (2009). Factores determinantes de la calidad de los servicios bancarios en un campus universitario venezolano. Investigación y Ciencia (45), 50-55.

Parasuraman, A., Zeithaml, V., y Berry, L. (1985). A Conceptual Model of Service Quality and Its Implications for Future Research. Journal of Marketing (49 (4) ), 41-50.

Parasuraman, A., Zeithaml, V., y Berry, L. (1988). SERVQUAL: A Multiple-Item Scale for Measuring Customer Perceptions of Service Quality. Journal of Retailing (64), 12-40.

Payne, A. (1996). La esencia de la mercadotecnia de servicios. México: Prentice Hall.

Rebolloso, E., Salvador, C., Fernández, B., y Cantón, P. (2004). Análisis y ampliación del SERVOUAL en los servicios universitarios. Revista de Psicología del
Trabajo y de las Organizaciones, XX (3), 355-373.

Rosander, A. C. (1992). La búsqueda de la calidad en los servicios. Madrid: Díaz de Santos.

Salazar, W., y Cabrera, M. (julio-diciembre de 2016). Diagnóstico de la calidad de servicio, en la atención al cliente, en la Universidad Nacional de Chimborazo Ecuador: Industrial Data, XIX (02), 1320.

Sampieri, R. H., y Collado, C. F. (2010). Metodología de la Investigación. México D.F.: McGraw-Hill / Interamericana Editores, S.A. DE C.V.

Summers, D. (2006). Administración de la calidad. México: Pearson Educación.

Zeithaml, V., y Parasuraman, A. y. (1985). Problems and Strategies in Services Marketing. Journal of Marketing (49 primavera), 33-45.

Zeithaml, V., Parasuraman, A., y Berry, L. (1993). Calidad Total en la gestión de servicios. Madrid: Díaz de Santos.

Zeithaml, V., Parasuraman, H., y Berry, L. (1990). Delivering Quality Service: Balancing customer perceptions and expectations. McGraw Hill. 